\title{
Women and Cardiovascular Disease - Pregnancy, the forgotten risk factor
}

Clare Arnott ${ }^{1-3}$, Sanjay Patel ${ }^{1,2}$, Jon Hyett ${ }^{2,4}$, Garry Jennings ${ }^{5}$, Mark Woodward ${ }^{3,6,7}$, David S. Celermajer ${ }^{1,2}$

${ }^{1}$ Department of Cardiology, Royal Prince Alfred Hospital, Sydney, Australia.

${ }^{2}$ Sydney Medical School, Sydney Australia.

${ }^{3}$ The George Institute of Global Health, University of New South Wales, Sydney, Australia.

${ }^{4}$ Department of Obstetrics, Royal Prince Alfred Hospital, Sydney, Australia.

${ }^{5}$ The National Heart Failure of Australia

${ }^{6}$ The George Institute for Global Health, University of Oxford, Oxford, UK

${ }^{7}$ Department of Epidemiology, Johns Hopkins University, Baltimore MD, USA

Corresponding author: Dr Clare Arnott; Department of Cardiology, Royal Prince Alfred Hospital, Sydney, Australia. Phone: 61295156111

Word Count: 3056 


\begin{abstract}
Cardiovascular disease (CVD) is the leading cause of death and disability in women globally, accounting for $32 \%$ of deaths in females. There are several female-specific risk factors for CVD that are under appreciated clinically, insufficiently researched and not given adequate attention in CVD risk prediction.
\end{abstract}

Hypertensive and metabolic disorders of pregnancy are independent risk factors for the development of premature CVD. They confer more than double the risk of CVD in exposed women, but are not included in any current multivariable CVD risk prediction models. Failure to recognise this risk leads to a lost opportunity to identify at risk women, institute primary preventive strategies, and potentially improve their health trajectory. This also translates into a missed opportunity to educate women and their families about their CVD risks, and to a lack of awareness and prioritisation of CVD within the broader community. Improving CVD outcomes for women globally also requires attention to research methodology and analysis. Researchers should be encouraged to include a thorough pregnancy history in prospective CVD datasets and to power their studies to report on gender disaggregated CVD outcomes. Particular attention should be given to inclusion of young women of child-bearing age in CVD intervention trials.

Ultimately, women should be offered CVD assessment using gender specific risk prediction models that are validated across broad ethic and socioeconomic groups. These prediction model should account for female specific risk factors and their complex interactions with traditional risk factors. This will pave the way for a gender-specific approach to CVD diagnosis, investigation and management. 
Key Words: pregnancy, pre-eclampsia, cardiovascular disease, risk calculators 


\section{Introduction}

Whilst traditionally considered a predominantly 'male’ disease, cardiovascular disease (CVD) is the leading cause of death in women worldwide, including in many low- and middle-income countries. In 2017, CVD accounted for 32\% of deaths in US women (>450 000) and a similar percentage worldwide[1]. CVD also ranked first globally, in 2017, in terms of disability adjusted life years amongst women. Furthermore, women with CVD are more likely to present later, experience more complications and worse outcomes than males[2-4]. Hence CVD is important in terms of lost life, sickness, health care expenditure, and lost economic productivity in women (as well as in men).

It is becoming more evident that there are several female-specific risk factors for the development of CVD[5]. (Figure 1) These include hypertensive and metabolic disorders of pregnancy which have been demonstrated, in large population studies, to be independent risk factors for the development of premature CVD[6-12]. In spite of this knowledge, pregnancy conditions, or indeed most female-specific risk factors, are not included in any of the widely used or nationally endorsed multivariable CVD risk calculators. Evidence suggests that, whilst CVD incidence is generally decreasing, the same positive trend is not being seen consistently in incidence and mortality rates in young female populations ( $<55$ years)[13]. Thus, a closer focus needs to be placed on a gender-specific approach to clinical care, research and translation in CVD. Moreover, any attempt to improve primary prevention and reduce CVD incidence and mortality in females needs to start with improving and refining the tools we use for risk stratification.

\section{Pregnancy and its Link to Cardiovascular Disease}


In population terms, women live longer than men and premenopausal women develop CVD approximately a decade later than males. Hence, a pre-menopausal woman aged 40 years has a similar risk to a man aged 30 years. Thus, whilst CVD is the leading cause of death in all adult female age categories[4, 14$]$, in early life the absolute rates of severe CVD in woman are quite low[15]. There are, however, certain groups with traditional risk factors at particuarly elevated risk, such as pre-menopausal women with hypertension[4, 14].

Additionally, women who develop hypertensive or metabolic disorders during pregnancy represent a key group with a proven, but clinically under-recognised, risk of developing premature CVD disease[7]. Such conditions include, but are not limited to; gestational hypertension (GH), pre-eclampsia (PE), eclampsia and gestational diabetes (GDM). PE is defined as new onset of hypertension after 20 weeks' gestation with evidence of proteinuria and/or acute kidney injury, liver dysfunction, neurological involvment, thrombocyotopaenia, haemolysis or foetal growth restriction. GH is defined as de novo hypertension after 20 weeks' gestation without any of the additonal features seen in PE[16]. GDM is defined as glucose intolerance diagnosed during pregnancy, with specific plasma glucose diagnostic criteria (fasting, 1 and 2 hour) revised and tightened by the American Diabetes Association in 2010 and the World Health Organisation in 2013[17].

In the US, recent estimates indicate that hypertensive disorders of pregnancy (HDP) are increasing in incidence, affecting approximately 9\% of pregnancies; and GDM inidence is also steadily increasing with current rates up to $9 \%[18,19]$. Globally, estimates suggest that 300 million women have experienced a pregnancy complicated by PE, and are at long term CVD risk[7]. 
Whilst it is well recognised that those with HDP share many of the same risk factors which pertain to CVD, such as obesity and age, what is poorly recognised is that these high risk pregnancies also represent an independent maternal CVD risk[7]. A wealth of scientific work, across a wide range of study designs, study populations, illness severities, maternal age and follow-up periods, independently links HDP, particularly PE, with poorer long term CVD outcomes, although the underlying pathophysiology is not completely understood. These studies quote hazard ratios for chronic hypertension of 1.88-6.7, 1.7-2.3 for ischaemic heart disease, 1.5-8.12 for CVD related death and 1.7-3.6 for stroke or stroke related death[6-12]. The most recent systematic review and meta-analysis of the relationship between PE and CVD, published in 2017, reported on 22 studies (18 cohort and 4 cross-sectional studies) of greater than 6.4 million women (>258 000 with PE). PE was independently associated with the later development of ischaemic heart disease (RR 2.50, 95\% CI 1.43-4.37), heart failure (RR 4.19, 95\% CI 2.09-8.38), CVD death (RR 2.21, 95\% CI 1.83-2.66) and stroke (RR 1.81, 95\% CI $1.29-2.55)[20]$

Women who have suffered HDP are also more likely to experience a premature CVD event, up to a decade earlier than women without a pregnancy complication[6, 21]. There may also be a 'dose-dependent' relationship, with evidence suggesting that recurrent PE confers a greater CVD risk than a single exposure[22]. This, again, highlights the improtance of accurate risk prediction and risk factor modification in young female populations.

In the CHAMPS study, of $>1$ million women, those with PE had twice the risk of developing CVD compared to unaffected women (HR 2.0, 95\% CI 1.7-2.2). Those with PE and concomitant metabolic syndrome, however, had a 12 fold increased risk of CVD as compared to women with neither (HR 11.7, 95\% CI 4.9-28.3)[7, 10]. In this study, the co-existence of 
PE and poor fetal growth also resulted in signficantly increased maternal mortality hazard ratios, compared to $\mathrm{PE}$ alone. This suggests that not only is $\mathrm{PE}$ an independent CVD risk factor, but that it may work synergistically with other traditional risk factors. Certainly, it highlights the fact that stratifying risk for a woman without consideration of her obstetric history may under-estimate her risk, leading to a lost opportunity to institute timely and evidence-based primary preventive strategies.

As mentioned, metabolic complications of pregnancy, such as GDM, are also strong risk factors for subsequent cardio-metabolic diseases in women. International studies suggest that the cumulative incidence of type 2 diabetes is close to $50 \%$, following gestational diabetes, with the sharpest increase in incidence of the disease appearing to occur in the immediate 5-10 year period post-partum[23, 24]. Additionally, multiple studies have now demonstrated that women with a history of GDM have a markedly elevated risk of subsequent CVD. The Nurses' Health Study II, reported a 43\% greater risk of myocardial infarction or stroke in women post GDM as compared to their contemporaries[25]. The relationship between gestational diabetes and CVD has also been evaluated in a recent meta-analysis of 7 studies and greater than 3 million women. This reported a RR for ischaemic heart disease of 2.09 (95\% CI 1.56-2.80) and that for stroke of 1.25 (95\% CI 1.07-1.48), though note was made of a high level of heterogeneity in the studies[26].

The best indicators of future CVD are risk factors that are not modifiable; sex, age and previous CVD[27]. Given that HDP represent conditions characterised by endothelial dysfunction/vascular function in early adulthood, they too may represent strong markers of future events. Furthermore, this highlights the importance of the postpartum period as an opportunity to identify these women and accurately risk stratify them (or modify their risk with 
intervention). The challenge lies in translating the known relative risk into an estimate of absolute risk in an individual, and then using this knowledge to make evidence based decisions regarding lifestyle and therapeutic interventions. Indeed, in spite of the recommendations of many large studies that hypertensive and metabolic conditions should be considered when evaluating a women's future CVD risk, no tools currently exist to allow clinicians to adequately achieve this[8, 28, 29].

\section{Barriers to Early Diagnosis and Risk Stratification in Women}

Whilst CVD is a leading cause of death and disability in women worldwide; awareness, risk stratification and prompt gender-specific management lags behind the male population in many regards. Indeed, many women, particularly those in ethnic/racial minority groups, have poor awareness of their susceptibility to heart disease[30, 31]. This issue of awareness is particularly true in young female populations (25-34years) who report time constraints, stress and a general lower perception of risk/relevance to themselves[32].

Pre-menopausal women are vastly under-represented in the CVD research field. Previously there has been almost complete exclusion of women of childbearing age from interventionbased CVD trials (particularly pharmacotherapeutic trials)[33-36]. Although some positive steps forward have been made, including the National Institute of Health (NIH) initiative in 1993 that all NIH funded clinical trials perform sex-specific analyses[37], these changes are yet to translate into the domain of clinical care in a meaningful way and inform evidence-based change in CVD risk stratification in young females who possess female-specific risk factors for premature CVD. 
Another important barrier to timely diagnosis of CVD in women is the fact that the 'face of CVD' is different in women and in men. The Women's Ischemia Syndrome Evaluation (WISE) Study showed that disease processes, response to treatment and outcomes are different in female populations[4, 14, 38]. Indeed, women have an apparent preponderance of inflammation as evidenced by greater high-sensitivity CRP (hsCRP) levels than men. Further, they are less likely to have obstructive coronary disease (CAD) on angiography, and are more likely to suffer from microvascular disease or endothelial dysfunction, both of which are difficult to diagnose using traditional 'chest pain’ pathways[4, 14]. Additionally, the way in which 'traditional' risk factors such as hypertension and lipid metabolism interact with novel risk factors is also different in females[39]. Thus, the use of traditional multivariable risk models that have not been validated in young female populations, particularly those who have experienced a HDP, is fraught with potential error and inaccuracy.

\section{Current risk calculators and their relevance to young female populations}

The great majority of women who experience a HDP will return to being normotensive in the post-partum period. As stated, however, this does not correlate to a return to 'baseline' CVD risk. Evidence suggests that only 1 in 5 women who experience a form of hypertension in pregnancy will have chronically elevated blood pressure and require treatment at the 2 year point[40]. In the long term, the risk of developing chronic hypertension in this population however is in the order of 4 fold that of the general female population (hazard ratios in different populations quoted in the range of 1.88-6.7)[6-12, 40]. Importantly, however, the link between HDP and CVD is not always via the development of chronic hypertension. That is, these women are at elevated risk of ischaemic heart disease independent of whether they develop chronic hypertension. It is particularly those women who remain normotensive that are likely 
to be under-estimated in any current multivariable risk calculator model, given the absence of 'traditional' risk factors.

Clinical trial data suggest that the treatment benefit of risk factor lowering in an individual is directly related to their initial CVD risk assessment score rather than any of their absolute initial risk factor values. Rather than targeting risk factors in isolation, an approach that focuses on overall risk can achieve lower clinical event rates[27, 41]. Thus, the focus of clinicians should be to identify and target all high risk individuals rather than to treat an individual parameter whilst ignoring the proven continuous dose-response relationship of these parameters with ultimate CVD risk[27, 42]. To make this change requires a CVD risk score that identifies those at high risk, however that may be defined in a specific jurisdiction. It is well recognised that the landmark Framingham risk score[43] was instrumental in changing the way we risk stratify for CVD incidence worldwide. This has influenced public health policy and primary preventative strategies on a global level[44] and resulted in a significant generational reduction in CVD disease[4, 15]. The CVD prediction models in current use (e.g. the Pooled Cohort Equation[45], GLOBORISK[46] and ASSIGN[47]) are Framingham-based, but none included young women in their derivation cohort(s). Hence we cannot accurately assess the absolute risk of CVD in young women using currently available tools and guidelines. Additionally, it is not clear from the current evidence in what manner hypertensive and metabolic complications of pregnancy interact with traditional risk factors to determine CVD risk[48].

More specifically, none of the CVD risk scores have been validated in a post PE cohort. The limitations of relying on a Framingham-based risk prediction are demonstrated in a study performed in the Netherlands which demonstrated similar CVD risk scores in post PE women who were currently normotensive and women who had uncomplicated pregnancies[28]. This 
has been extrapolated to suggest possible comparable long-term CVD risk in these women, in spite of the known independent risk that PE confers, as evidenced by large population studies[7, 10]. Such conclusions highlight the need for a risk prediction model that independently accounts for pregnancy-associated risk, such that at-risk women are not incorrectly assigned into a lower CVD risk category and thereby not offered primary preventative therapies. In slight disagreement with the Dutch study, several studies have reported that those with a history of HDP, particularly PE, have an elevated Framingham risk score, compared to females who experienced uncomplicated pregnancies[28, 29, 49, 50]. This is hardly surprising giving that HDP and CVD share common risk factors, such as obesity and advanced age. A British study, that evaluated risk prediction in women who had experienced a metabolic or hypertensive pregnancy complication, evaluated risk using a 10-year Framingham-based CVD risk score at 18 years post-pregnancy in 3416 women[49]. Both GH and PE were associated with a greater number of CVD risk factors, such as increased body mass index (BMI), blood pressure and fasting lipids; with 10 year Framingham risk score of 5.09\% in PE and 3.55\% in those with no HDP.

These studies pave the way for the next phase of research, whereby pregnancy complications will be recognised as independent risk factors and considered in primary prevention strategies. An understanding of the complex interplay between 'traditional' risk factors and pregnancy/reproductive ones will facilitate accurate risk prediction.

To date, the only internationally recognised, though not widely used, female-specific CVD risk prediction model is The Reynolds Risk Score[51]. It was devised with the specific aim of improving the predictive power of CVD risk calculators in female populations through consideration of female-specific factors. The authors justified the need for this algorithm on 
previous studies that suggested that at least $20 \%$ of women who experienced a major CVD event did not possess a 'traditional' risk factor (hypertension, hyperlipidaemia, diabetes, smoking) and thus were likely being inaccurately risk stratified and not offered adequate primary intervention[52]. They proposed two new prediction models using a composite of inflammatory and lipid biomarkers, such as hsCRP and lipoprotein-a, in additional to traditional risk factors. The algorithms were tested in a cohort of almost 25000 women ( $>45$ years) from the Women's Heart Study, with around a decade of follow-up for major CVD events or death. 'Model B', the more simplified and clinically applicable of the proposed algorithms, demonstrated improved accuracy in risk stratifying women as compared to the Adult Treatment Panel III (ATP-III) prediction model. This study reported on a predominantly white population, and did not account for hormonal or reproductive factors. Furthermore, the Reynolds Risk Score was found to under-estimate risk in women by 21\% in the Multi-Ethnic Study of Atherosclerosis (MESA) population[53]. This work was followed closely by a Reynold's Risk Score for men[54], which demonstrated that hsCRP (and parental history) potentially improved the cardiovascular predictive power as compared to traditional models in men as well as women. This supports a general importance for hsCRP in CVD risk rather than as an indicator of female-specific risk.

To some extent, the poor performance of current CVD risk scores can be attributed to their derivation from historical data, in which CVD events rates cannot reasonably be expected to calibrate well with current CVD rates, leading to over-estimation because 'background' risks of CVD have decreased in recent years, in many populations. However, their discrimination between those who will, and will not, go on to have a CVD event is also sub-optimal. Improved scores, which include non-traditional, but important, gender-specific risk factors are required. 
Ideally, such scores should be applied, whenever possible, after recalibration to contemporary circumstances.

\section{The Way Forward}

The authors suggest that now is the time to give appropriate attention to the impact of gender with respect to the pathophysiology of CVD, response to therapies, as well as risk prediction and primary prevention. We would like to add our voice to the call to action on gender in CVD that is gaining traction globally. We challenge researchers to ensure their studies are powered to report gender-specific outcomes and include comprehensive pregnancy-related variables which are analysed for independent links with adverse CVD outcomes. Lastly, we ask researchers to consider how they can assess CVD risk in female populations, with a high degree of accuracy across all ethnic groups.

From a clinical perspective, cardiologists and general practitioners need to routinely include an obstetric history as part of their cardiovascular risk assessment in all women, regardless of their age. Prospective management of women following pregnancy, however, is a clinical challenge due to a lack of robust randomised data. Those who have experienced a hypertensive disorder of pregnancy, particularly when accompanied by gestational diabetes, metabolic syndrome or foetal growth restriction, should be monitored closely for the development of CVD. In the absence of global consensus in guidelines with respect to timing or nature of cardiovascular follow-up in this group [55, 56], expert opinion suggests review initially at 6 to 12 weeks post-partum and then at 6 to 12 months.[57] This cardiovascular assessment should include blood pressure assessment and review of other modifiable risk factors, such as weight management, exercise and smoking cessation.[58] Thereafter, frequency of follow-up should be 1 to 5 yearly, depending on an individualised assessment of risk. The routine use of low 
dose aspirin, statins or other pharmacotherapy is not currently recommended, in the absence of another indication.

A high index of suspicion should be used when assessing a women, regardless of age, who presents with chest pain or anginal-equivalent symptoms on the background of a hypertensive or metabolic disorder of pregnancy. Whilst prospective data is currently lacking, the authors suggest early referral to a cardiologist and consideration of an objective investigation, such as stress echocardiography.

As long as CVD continues to claim the lives of a significant proportion of women worldwide, and women demonstrate poorer in-hospital outcomes with greater complications[59], assessment of female-specific risk factors needs to be prioritised. To this end, there is urgent need for a multivariable CVD risk calculator that independently accounts for hypertensive and metabolic disorders of pregnancy, ideally using data collected from diverse populations of women that are representative of wider communities.

\section{Acknowledgments}

None. 


\section{Figure Legend}

Figure 1: Cardiovascular risk in women

Traditional and female-specific factors related to the risk of cardiovascular disease in women. 


\section{References}

[1] (IHME). IfHMaE. GBD Compare Data Visualization. Seattle, WA: IHME, University of Washington, 2016. Available from http:// vizhubhealthdataorg/gbd-compare. (Accessed 2018).

[2] Mehta LS, Beckie TM, DeVon HA, Grines CL, Krumholz HM, Johnson MN, et al. Acute Myocardial Infarction in Women: A Scientific Statement From the American Heart Association. Circulation. 2016;133:916-47.

[3] Dreyer RP, Beltrame JF, Tavella R, Air T, Hoffmann B, Pati PK, et al. Evaluation of gender differences in Door-to-Balloon time in ST-elevation myocardial infarction. Heart Lung Circ. 2013;22:861-9.

[4] Shaw LJ, Bairey Merz CN, Pepine CJ, Reis SE, Bittner V, Kelsey SF, et al. Insights from the NHLBI-Sponsored Women's Ischemia Syndrome Evaluation (WISE) Study: Part I: gender differences in traditional and novel risk factors, symptom evaluation, and genderoptimized diagnostic strategies. J Am Coll Cardiol. 2006;47:S4-S20.

[5] Peters SA, Woodward M. Women's reproductive factors and incident cardiovascular disease in the UK Biobank. Heart. 2018.

[6] McDonald SD, Malinowski A, Zhou Q, Yusuf S, Devereaux PJ. Cardiovascular sequelae of preeclampsia/eclampsia: a systematic review and meta-analyses. Am Heart J. 2008;156:918-30.

[7] Ahmed R, Dunford J, Mehran R, Robson S, Kunadian V. Pre-eclampsia and future cardiovascular risk among women: a review. J Am Coll Cardiol. 2014;63:1815-22.

[8] Bellamy L, Casas JP, Hingorani AD, Williams DJ. Pre-eclampsia and risk of cardiovascular disease and cancer in later life: systematic review and meta-analysis. BMJ. 2007;335:974.

[9] Brown MC, Best KE, Pearce MS, Waugh J, Robson SC, Bell R. Cardiovascular disease risk in women with pre-eclampsia: systematic review and meta-analysis. Eur J Epidemiol. 2013;28:1-19.

[10] Ray JG, Vermeulen MJ, Schull MJ, Redelmeier DA. Cardiovascular health after maternal placental syndromes (CHAMPS): population-based retrospective cohort study. Lancet. 2005;366:1797-803.

[11] Drost JT, Arpaci G, Ottervanger JP, de Boer MJ, van Eyck J, van der Schouw YT, et al. Cardiovascular risk factors in women 10 years post early preeclampsia: the Preeclampsia Risk EValuation in FEMales study (PREVFEM). Eur J Prev Cardiol. 2012;19:1138-44. [12] Riise HK, Sulo G, Tell GS, Igland J, Nygard O, Vollset SE, et al. Incident Coronary Heart Disease After Preeclampsia: Role of Reduced Fetal Growth, Preterm Delivery, and Parity. J Am Heart Assoc. 2017;6.

[13] Garcia M, Mulvagh SL, Merz CN, Buring JE, Manson JE. Cardiovascular Disease in Women: Clinical Perspectives. Circ Res. 2016;118:1273-93.

[14] Gierach GL, Johnson BD, Bairey Merz CN, Kelsey SF, Bittner V, Olson MB, et al. Hypertension, menopause, and coronary artery disease risk in the Women's Ischemia Syndrome Evaluation (WISE) Study. J Am Coll Cardiol. 2006;47:S50-8.

[15] Bots SH, Peters SAE, Woodward M. Sex differences in coronary heart disease and stroke mortality: a global assessment of the effect of ageing between 1980 and 2010. BMJ Glob Health. 2017;2:e000298.

[16] Brown MA, Magee LA, Kenny LC, Karumanchi SA, McCarthy FP, Saito S, et al. Hypertensive Disorders of Pregnancy: ISSHP Classification, Diagnosis, and Management Recommendations for International Practice. Hypertension. 2018;72:24-43.

[17] Goedegebure EAR, Koning SH, Hoogenberg K, Korteweg FJ, Lutgers HL, Diekman MJM, et al. Pregnancy outcomes in women with gestational diabetes mellitus diagnosed 
according to the WHO-2013 and WHO-1999 diagnostic criteria: a multicentre retrospective cohort study. BMC Pregnancy Childbirth. 2018;18:152.

[18] Zhang S, Wang L, Leng J, Liu H, Li W, Zhang T, et al. Hypertensive disorders of pregnancy in women with gestational diabetes mellitus on overweight status of their children. J Hum Hypertens. 2017;31:731-6.

[19] DeSisto CL, Kim SY, Sharma AJ. Prevalence estimates of gestational diabetes mellitus in the United States, Pregnancy Risk Assessment Monitoring System (PRAMS), 2007-2010. Prev Chronic Dis. 2014;11:E104.

[20] Wu P, Haththotuwa R, Kwok CS, Babu A, Kotronias RA, Rushton C, et al. Preeclampsia and Future Cardiovascular Health: A Systematic Review and Meta-Analysis. Circ Cardiovasc Qual Outcomes. 2017;10.

[21] Tooher J, Chiu CL, Yeung K, Lupton SJ, Thornton C, Makris A, et al. High blood pressure during pregnancy is associated with future cardiovascular disease: an observational cohort study. BMJ Open. 2013;3.

[22] Brouwers L, van der Meiden-van Roest AJ, Savelkoul C, Vogelvang TE, Lely AT, Franx A, et al. Recurrence of pre-eclampsia and the risk of future hypertension and cardiovascular disease: a systematic review and meta-analysis. BJOG. 2018;125:1642-54. [23] Kim C, Newton KM, Knopp RH. Gestational diabetes and the incidence of type 2 diabetes: a systematic review. Diabetes Care. 2002;25:1862-8.

[24] Schoenaker D, Mishra GD. Association Between Age at Menarche and Gestational Diabetes Mellitus: The Australian Longitudinal Study on Women's Health. Am J Epidemiol. 2017;185:554-61.

[25] Tobias DK, Stuart JJ, Li S, Chavarro J, Rimm EB, Rich-Edwards J, et al. Association of History of Gestational Diabetes With Long-term Cardiovascular Disease Risk in a Large Prospective Cohort of US Women. JAMA Intern Med. 2017;177:1735-42.

[26] Li J, Song C, Li C, Liu P, Sun Z, Yang X. Increased risk of cardiovascular disease in women with prior gestational diabetes: A systematic review and meta-analysis. Diabetes Res Clin Pract. 2018;140:324-38.

[27] Jackson R, Lawes CM, Bennett DA, Milne RJ, Rodgers A. Treatment with drugs to lower blood pressure and blood cholesterol based on an individual's absolute cardiovascular risk. Lancet. 2005;365:434-41.

[28] Breetveld NM, Ghossein-Doha C, van Kuijk S, van Dijk AP, van der Vlugt MJ, Heidema WM, et al. Cardiovascular disease risk is only elevated in hypertensive, formerly preeclamptic women. BJOG. 2015;122:1092-100.

[29] Hermes W, Tamsma JT, Grootendorst DC, Franx A, van der Post J, van Pampus MG, et al. Cardiovascular risk estimation in women with a history of hypertensive pregnancy disorders at term: a longitudinal follow-up study. BMC Pregnancy Childbirth. 2013;13:126. [30] Giardina EG, Sciacca RR, Flink LE, Bier ML, Paul TK, Moise N. Cardiovascular disease knowledge and weight perception among Hispanic and non-Hispanic white women. $\mathrm{J}$ Womens Health (Larchmt). 2013;22:1009-15.

[31] Flink LE, Sciacca RR, Bier ML, Rodriguez J, Giardina EG. Women at risk for cardiovascular disease lack knowledge of heart attack symptoms. Clin Cardiol. 2013;36:1338.

[32] Mosca L, Hammond G, Mochari-Greenberger H, Towfighi A, Albert MA, American Heart Association Cardiovascular D, et al. Fifteen-year trends in awareness of heart disease in women: results of a 2012 American Heart Association national survey. Circulation. 2013;127:1254-63, e1-29.

[33] Dougherty AH. Gender balance in cardiovascular research: importance to women's health. Tex Heart Inst J. 2011;38:148-50. 
[34] Harris DJ, Douglas PS. Enrollment of women in cardiovascular clinical trials funded by the National Heart, Lung, and Blood Institute. N Engl J Med. 2000;343:475-80.

[35] Kim ES, Menon V. Status of women in cardiovascular clinical trials. Arterioscler Thromb Vasc Biol. 2009;29:279-83.

[36] Melloni C, Berger JS, Wang TY, Gunes F, Stebbins A, Pieper KS, et al. Representation of women in randomized clinical trials of cardiovascular disease prevention. Circ Cardiovasc Qual Outcomes. 2010;3:135-42.

[37] Lundberg GP, Mehta LS, Volgman AS. Specialized Care for Women: the Impact of Women's Heart Centers. Curr Treat Options Cardiovasc Med. 2018;20:76.

[38] Bairey Merz CN, Shaw LJ, Reis SE, Bittner V, Kelsey SF, Olson M, et al. Insights from the NHLBI-Sponsored Women's Ischemia Syndrome Evaluation (WISE) Study: Part II: gender differences in presentation, diagnosis, and outcome with regard to gender-based pathophysiology of atherosclerosis and macrovascular and microvascular coronary disease. $\mathrm{J}$ Am Coll Cardiol. 2006;47:S21-9.

[39] Matthews KA, Kuller LH, Sutton-Tyrrell K, Chang YF. Changes in cardiovascular risk factors during the perimenopause and postmenopause and carotid artery atherosclerosis in healthy women. Stroke. 2001;32:1104-11.

[40] Bramham K, Nelson-Piercy C, Brown MJ, Chappell LC. Postpartum management of hypertension. BMJ. 2013;346:f894.

[41] Anderson KM, Odell PM, Wilson PW, Kannel WB. Cardiovascular disease risk profiles. Am Heart J. 1991;121:293-8.

[42] Law MR, Wald NJ. Risk factor thresholds: their existence under scrutiny. BMJ. 2002;324:1570-6.

[43] D'Agostino RB, Sr., Vasan RS, Pencina MJ, Wolf PA, Cobain M, Massaro JM, et al. General cardiovascular risk profile for use in primary care: the Framingham Heart Study. Circulation. 2008;117:743-53.

[44] Board JBS. Joint British Societies' consensus recommendations for the prevention of cardiovascular disease (JBS3). Heart. 2014;100 Suppl 2:ii1-ii67.

[45] Goff DC, Jr., Lloyd-Jones DM, Bennett G, Coady S, D'Agostino RB, Gibbons R, et al. 2013 ACC/AHA guideline on the assessment of cardiovascular risk: a report of the American College of Cardiology/American Heart Association Task Force on Practice Guidelines. Circulation. 2014;129:S49-73.

[46] Ueda P, Woodward M, Lu Y, Hajifathalian K, Al-Wotayan R, Aguilar-Salinas CA, et al. Laboratory-based and office-based risk scores and charts to predict 10-year risk of cardiovascular disease in 182 countries: a pooled analysis of prospective cohorts and health surveys. Lancet Diabetes Endocrinol. 2017;5:196-213.

[47] Woodward M, Brindle P, Tunstall-Pedoe H, estimation Sgor. Adding social deprivation and family history to cardiovascular risk assessment: the ASSIGN score from the Scottish Heart Health Extended Cohort (SHHEC). Heart. 2007;93:172-6.

[48] Tsao CW, Vasan RS. Cohort Profile: The Framingham Heart Study (FHS): overview of milestones in cardiovascular epidemiology. Int J Epidemiol. 2015;44:1800-13.

[49] Fraser A, Nelson SM, Macdonald-Wallis C, Cherry L, Butler E, Sattar N, et al. Associations of pregnancy complications with calculated cardiovascular disease risk and cardiovascular risk factors in middle age: the Avon Longitudinal Study of Parents and Children. Circulation. 2012;125:1367-80.

[50] Smith GN, Pudwell J, Walker M, Wen SW. Ten-year, thirty-year, and lifetime cardiovascular disease risk estimates following a pregnancy complicated by preeclampsia. J Obstet Gynaecol Can. 2012;34:830-5. 
[51] Ridker PM, Buring JE, Rifai N, Cook NR. Development and validation of improved algorithms for the assessment of global cardiovascular risk in women: the Reynolds Risk Score. JAMA. 2007;297:611-9.

[52] Khot UN, Khot MB, Bajzer CT, Sapp SK, Ohman EM, Brener SJ, et al. Prevalence of conventional risk factors in patients with coronary heart disease. JAMA. 2003;290:898-904. [53] DeFilippis AP, Young R, Carrubba CJ, McEvoy JW, Budoff MJ, Blumenthal RS, et al. An analysis of calibration and discrimination among multiple cardiovascular risk scores in a modern multiethnic cohort. Ann Intern Med. 2015;162:266-75.

[54] Ridker PM, Paynter NP, Rifai N, Gaziano JM, Cook NR. C-reactive protein and parental history improve global cardiovascular risk prediction: the Reynolds Risk Score for men. Circulation. 2008;118:2243-51, 4p following 51.

[55] Thilaganathan B, Kalafat E. Cardiovascular System in Preeclampsia and Beyond. Hypertension. 2019;73:522-31.

[56] Groenhof TKJ, van Rijn BB, Franx A, Roeters van Lennep JE, Bots ML, Lely AT. Preventing cardiovascular disease after hypertensive disorders of pregnancy: Searching for the how and when. Eur J Prev Cardiol. 2017;24:1735-45.

[57] Staff AC, Redman CW, Williams D, Leeson P, Moe K, Thilaganathan B, et al. Pregnancy and Long-Term Maternal Cardiovascular Health: Progress Through Harmonization of Research Cohorts and Biobanks. Hypertension. 2016;67:251-60. [58] Spaan J, Peeters L, Spaanderman M, Brown M. Cardiovascular risk management after a hypertensive disorder of pregnancy. Hypertension. 2012;60:1368-73.

[59] Khan E, Brieger D, Amerena J, Atherton JJ, Chew DP, Farshid A, et al. Differences in management and outcomes for men and women with ST-elevation myocardial infarction.

Med J Aust. 2018;209:118-23. 